\title{
Mindful Parenting in Mental Health Care
}

\author{
Susan M. Bögels • Annukka Lehtonen • Kathleen Restifo
}

Published online: 25 May 2010

(C) The Author(s) 2010. This article is published with open access at Springerlink.com

\begin{abstract}
Mindfulness is a form of meditation based on the Buddhist tradition, which has been used over the last two decades to successfully treat a multitude of mental health problems. Bringing mindfulness into parenting ("mindful parenting") is one of the applications of mindfulness. Mindful parenting interventions are increasingly being used to help prevent and treat mental disorders in children, parenting problems, and prevent intergenerational transmission of mental disorders from parents to children. However, to date, few studies have examined the hypothesized mechanisms of change brought about by mindful parenting. We discuss six possible mechanisms through which mindful parenting may bring about change in parent-child interactions in the context of child and parent mental health problems. These mechanisms are hypothesized to be mediated by the effects of mindfulness on parental attention by: (1) reducing parental stress and resulting parental reactivity; (2) reducing parental preoccupation resulting from parental and/or child psychopathology; (3) improving parental executive functioning in impulsive parents; (4) breaking the cycle of intergenerational transmission of
\end{abstract}

S. M. Bögels ( $\bowtie)$

Research Institute of Child Development and Education,

University of Amsterdam,

Nieuwe Prinsengracht 130,

1018 VZ, Amsterdam, The Netherlands

e-mail: S.M.Bogels@uva.nl

\section{A. Lehtonen}

Department of Psychiatry, University of Oxford,

Oxford, UK

K. Restifo

Department of Clinical Psychological Science,

Maastricht University,

Maastricht, The Netherlands dysfunctional parenting schemas and habits; (5) increasing self-nourishing attention; and (6) improving marital functioning and co-parenting. We review research that has applied mindful parenting in mental health settings, with a focus on evidence for these six mechanisms. Finally, we discuss directions for future research into mindful parenting and the crucial questions that this research should strive to answer.

Keywords Mindfulness · Parenting ·

Child mental disorder. Parent mental disorder - Attention .

Mindful parenting

\section{Introduction}

Mindfulness is a form of meditation based on the Buddhist tradition. Over the last two decades, mindfulness-based interventions have been used to successfully treat a multitude of mental health problems. Bringing mindfulness into parenting ("mindful parenting") is one of the newer applications of mindfulness in mental health contexts. Mindful parenting has been defined by Kabat-Zinn and Kabat-Zinn (1997) as: "paying attention to your child and your parenting in a particular way: intentionally, here and now, and non-judgementally". Several papers have addressed the role of mindful parenting in mental health problem prevention and treatment contexts on a theoretical, descriptive practical, and/or empirical outcome level (e.g., Altmaier and Maloney 2007; Bögels et al. 2008; Dumas 2005; Duncan et al. 2009; Sawyer 2007; Singh et al. 2007; Wahler et al. 2008). However, less attention has been paid to the underlying mechanisms of change, particularly in the context of mental health problems in parents and/or children. Our goal is to review the mindful parenting 
literature from the perspective of issues involved in why mindful parenting may work in the context of child and parent mental health problems. Before discussing these issues, we first review research into the effects of mindfulness on attention, in general. And before that, we will first shortly address the effects of mindfulness-based treatment approaches to adult mental health problems. We conclude the paper with directions of research into the effectiveness of mindful parenting in general and into the issues involved in change through mindful parenting in particular.

\section{How Has Mindfulness Been Applied in Therapies?}

Kabat-Zinn (1990) developed the mindfulness-based stress reduction (MBSR) program for trying to help chronically ill people to cope with their illness. MBSR has been helpful for a variety of physical and psychological conditions. Grossman et al. (2004) did a meta-analysis on the (mental) health benefits of MBSR programs and consistently found respectable effect sizes for MBSR interventions. This was also the case for studies that included control treatments, which would suggest a benefit specific for mindfulness training. Similar findings were reported by Baer (2003).

Mindfulness meditation is the basis of mindfulnessbased cognitive therapy (MBCT), a treatment approach developed specifically for depressed patients (Segal et al. 2002). MBCT targets the patterns of thinking activated by dysphoria: the association between low mood and negative, repetitive thinking that is characteristic of major depression. This kind of thinking is still assumed to be typical for patients recovered from depression, who can be vulnerable to subsequent periods of low mood, as this may re-activate the negative cognitions. The aim of the intervention is to enable participants to decenter or take distance from their negative thinking and therefore interrupt the cycle of negative, repetitive thoughts before they escalate into fullblown depressive episode again.

Several studies have demonstrated the effectiveness of the MBCT program in decreasing the likelihood of another depressive episode among patients with more than three episodes of depression (e.g., Ma and Teasdale 2004; Teasdale et al. 2001). Note that ruminative thinking has also been found characteristic for many other mental disorders, such as anxiety and eating disorders, and thus, mindfulness training could be beneficial for those disorders as well. In line, mindfulness-based approaches have been found effective in mental disorders in which rumination is a central feature, such as generalized anxiety disorder (Roemer et al. 2008), social anxiety disorder (Bögels et al. 2006), and eating disorders (Kristeller et al. 2006). In addition to the MBSR and the MBCT, other empirically supported intervention programs have included mindfulness training as one of the components involved [dialectical behavior therapy, Linehan (1993) and acceptance and commitment therapy, Hayes et al. (1999)].

\section{Attention and Mindfulness}

Before understanding possible working mechanisms of mindfulness for improving parenting in mental health contexts, we focus on understanding change through mindfulness in general. Attentional processes may be one of the key mechanisms underlying change in mindfulness. Bishop (2002) pointed out that the concepts involved in mindfulness - being present in the moment, focussing on the reality and accepting it for what it is-all involve different aspects of attention. This is consistent with the argument that, in general, meditation modifies attention, as highlighted by for example Valentine and Sweet (1999), Brown and Ryan (2003) and Sethi (1989), and by Cahn and Polich (2006) in the context of different brain imaging studies. However, despite the general connection that has been drawn between attention and meditation, there has not been much research investigating the specific ways in which mindfulness meditation affects attention. It is not possible to provide a comprehensive overview of all the available studies in this paper, but we will mention a few studies that demonstrate the role of attention in mindfulness.

Mindfulness has been shown to improve executive attention in general. Using the Attention Network Test, which requires participants to respond to an arrow target, flanked by distractors that point either to the same or different direction as the arrow, Jha et al. (2007) found improvements in participants' performance depending on their meditation experience. The results suggested that, to begin with at baseline, participants with prior experience in meditation had better conflict monitoring skills (ability to prioritize between conflicting responses and tasks) than participants with no experience and that taking part in the MBSR course improved orienting skills. Jha et al. (2007) argued that previous or newly gained meditation experience improves voluntary top-down attention control. Tang et al. (2007) had an experimental group do 5 days of integrative body-mind training (including mindfulness meditation as one component) while a control group was assigned to relaxation training. Again, the Attention Network Test was used to measure attention abilities before and after training. Like Jha et al. (2007), Tang et al. found that the group trained in meditation did better in conflict monitoring after training, while there was no effect on the control group who received relaxation training. However, the training had no effect on orienting or alerting skills, and Tang et al. (2007) suggested that short-term meditation practice improves executive attention in particular. 
The effect of mindfulness training on participants' performance in attention interference tasks has also been examined. Ortner et al. (2007) tested participants who differed in the length of their meditation experience and found that the longer a participant had been meditating, the less emotional interference they showed. The task required participants to categorize tones while being presented with neutral, pleasant, or unpleasant pictures. The effects of meditation experience emerged both for pleasant and unpleasant pictures. Ortner et al. (2007) further showed that when meditation-naïve participants were assigned to mindfulness meditation, relaxation meditation training, or waitlist control, after 7-week training, only the mindfulness meditation group showed a reduction in emotional interference, that is, they were quicker to disengage their attention from emotional stimuli. Furthermore, the mindfulness group was the only one to report decreased intensity of feelings after seeing the unpleasant pictures. Wenk-Sormaz (2005) used the Stroop paradigm and showed that participants in mindfulness sitting meditation group showed less interference in the Stroop task than participants in the control group. Finally, Valentine and Sweet (1999) found that both mindfulness and concentrative meditators did better on a sustained attention task than controls, and mindfulness meditators were better than the other groups when the stimuli were unexpected, suggesting that they were less susceptible to the finding that focussed attention is impaired with unexpected stimuli.

The studies discussed above suggest that attention is one of the cognitive processes influenced by mindfulness. In particular, the ability to disengage from unexpected and emotional stimuli and attention conflict monitoring improve as a result of meditation practice. It is important to note that this research is still very much at the beginning stage. The results are often inconclusive because the tasks used to measure attention, the interventions introduced as mindfulness practices, and the groups tested vary from one study to another. The studies have also thus far largely failed to do longer-term follow-up, so it is impossible to know whether the effects on attention are long-lasting or not. Consequently, it is not possible to determine at this point in time how exactly mindfulness affects attention.

\section{Why Try Mindfulness for Improving Parenting?}

When considering mindfulness and how it might suit addressing issues central in parenting, in the context of parent and child mental health problems, there are several issues that need to be taken into account. They all involve the role of attention. We hypothesize that mindfulnessbased parenting interventions may exert their effects by targeting six domains: (1) parental stress; (2) parental preoccupation resulting from parental and/or child psychopathology; (3) parental executive functioning; (4) repeating dysfunctional own upbringing schemes and habits; (5) self-nourishing attention; and (6) marital functioning and co-parenting. Improvements in these six domains may positively affect the parent-child relationship and parenting skills.

\section{Parenting Stress}

The first hypothesis to be explored is that mindfulness training reduces parental stress, and thereby improves parenting. Parenting stress has been found to strongly affect parenting skills: under stress, parents become more rejecting, controlling, and reactive, and less warm towards their children (e.g., Belsky 1984; Webster-Stratton 1990). To illustrate, Crnic et al. (2005) found that, in a longitudinal study of 125 typically developing children, cumulative major life event stress and cumulative parenting daily hassles independently predicted less maternal positivity in interaction with their 5-year-old child. Cumulative stress resulting from parenting daily hassles (but not major life event stress) also predicted less dyadic parent-child pleasure. One explanation for the effect of parental stress on parenting was suggested by Siegel and Hartzell (2004). In stressful parenting situations (e.g., a child refusing to go to school and throwing him/herself on the ground), parents may fall back into a fight, flight, or freeze response which is activated under threat - the basic survival response. This fast response is a short route in the brain, located in the brainstem. The brainstem helps regulate aspects of alertness and the bodily functions such as breathing and heart rate. The brainstem is also responsible for our fight-flight-freeze response, combined with the limbic area (Siegel 2009). This short route in the brain does not involve the prefrontal cortex, which is important for paying attention. A survival response such as fighting is functional in situation of real danger, as it is fast (e.g., parent grasping the child's arm with force when the child is crossing a road full of traffic) but is ineffectual and potentially destructive for the parentchild relationship in cases where there is no real or immediate danger. A flight or freeze response may be seen in depressed or anxious parents, who withdraw from the child under stress. Parents who suffer from mental disorders, or who have children with mental disorders, are likely to be exposed to more stress (e.g., Baker-Ericzen et al. 2005).

It is interesting to note that parenting stress not only negatively affects parenting, but also negatively affects the perceived marital quality (Lavee et al. 1996). As marital quality, in turn, negatively affects parenting (discussed below in the section Marital Functioning and Co-parenting), there appears to be a direct and indirect way in which 
parental stress negatively affects parenting. Mindfulness training directly intervenes in parental stress and, as such, may be an alternative way of intervening in parenting skills, in addition to or as an alternative for parent training programs.

\section{Parental Preoccupation}

The second hypothesis to be explored is that mindfulness improves parenting by reducing parental preoccupation and/or negative bias. These have been widely documented in the context of postnatal depression, and an important factor involved in this process seems to be the way in which depression affects the parent's (in most existing studies, the mother's) sensitivity and, consequently, parentchild interaction (Goodman and Gotlib 2002; Murray et al. 1993, 1996; Restifo and Bögels 2009; Stein et al. 1991). Stein et al. (2009) suggested that attention plays an important part in this. They argued that because mothers' attention is taken up by their repetitive, negative, preoccupied thinking, which is characteristic to depression (Nolen-Hoeksema 1991, 2000), they have less attention to allocate to their child during interaction. Consequently, the mother is more likely to miss the child's cues, and the interaction is thus less sensitive and synchronized than interaction between well mothers and their children. Furthermore, Sawyer (2007) described mindfulness as a necessary prerequisite for the affective attunement that occurs within the intersubjective relatedness of mother and infant that occurs around the infant's seventh and ninth month. The "state-sharing" mother who is attuned to her child promotes the child's developing theory of mind (or understanding of others' mind states), by creating connectedness, and by the child feeling understood. Sawyer (2007) pointed to the potential role of mindfulness for depressed mothers in order to promote mental presence, both with the child's as well as her own needs. Also, in other mental disorders in which repetitive, preoccupied thinking plays a central role, such as anxiety disorders and eating disorders, Mindfulness training may improve parental emotional presence.

Parental preoccupation with ruminative negative thinking may not only reduce attention for the child and affect synchrony in interaction, it may also affect the focus of the attention for the child. To illustrate, a mother who is preoccupied by her own eating disorder or her own obsessive-compulsive focus on neatness may, while observing her child eating a first spoon of food, be preoccupied by the child spilling the food or eating in an uncontrolled way rather than noticing the child's experimenting with all the aspects of eating (e.g., motor, smell, taste, texture and digestive).

Mindfulness is hypothesized to break the cycle of repetitive, negative thoughts, and to allow parents, in interaction with their child, to attend to their child, rather than to their inner ruminations. Furthermore, mindfulness techniques may help parents to pay attention to their child in a more open, non-judgmental way, instead of having a biased attention for negative cues in the child or a biased (negative) interpretation of the child's behavior. Therefore, mindfulness applied to parents with mental disorders is hypothesized to improve parenting and the parent-child interaction and to prevent intergenerational transmission of negative attentional biases. Consistent with this hypothesis, a recent study showed that, in a sample of 121 depressed parents with 9-15 year-old children, the parent's level of mindfulness mediated the relationship between parents' current depressive symptoms and poor parenting (Roland 2009).

Parental preoccupation or negative attentional bias may result not only from the parental mental disorder, but also from the child's mental disorder. That is, if a child has a history of "bad" or "difficult" behavior, such as having tantrums, lying, uncontrollable negative emotions, resulting from a child's mental disorder or other child disability, the parent's attention may become preoccupied by the expected bad or difficult child behavior. Such attentional bias for negative child behavior interferes with parental attention for neutral or positive child behavior. Thus, it is likely that parents pay increasing attention to their children's problem behavior, become more impulsive and irritable as well as less attentive to the child in general and less patient. These behaviors come under the category of "parental reactivity", which Miller-Lewis et al. (2006) found to be the single significant predictor of chronic externalizing problems in 6year-old children. Dumas (2005), in the context of families of disruptive children, called such vicious circles of rigid patterns of disagreements and conflicts, which are overlearned with repeated practice, "automatized transactional procedures". In addition to parental reactivity, parental preoccupation or lack of parental mindfulness may have a negative effect on other parenting tasks and behaviors in parents of children with difficulties. To illustrate, fathers of children with intellectual disabilities who reported being more present-focused on a mindful parenting scale also reported more involvement in child-related parenting and roles related to child socialization (MacDonald et al. 2009).

Diagnostic labels may also hinder non-judgmental parental attention: for example, a diagnosis of an autismspectrum disorder, oppositional-defiant or conduct disorder, or ADHD may shape the way parents view their child. The diagnostic label may increase the parents' attentional bias to symptom behaviors which may set off a chain of negative cognitions. For example, when a child diagnosed with autism-spectrum disorder has a difficult play date, the mother focuses on what the child does wrong (attentional bias), labels it ("that's his autism causing it"), and then begins to ruminate ("he's abnormal," "he'll never be 
mainstreamed," "He'll never become independent," "I'll never have time for myself again," "I am a bad mother because I have not managed to change his behavior," "I should never have had children," "I cannot cope with this any longer"). The sadness and despair caused by such a negative chain of cognition may lead to dysfunctional parenting behaviors such as anger outbursts, withdrawal, or overcontrol in order not to feel the sadness and despair. Coyne and Wilson (2004) denoted such a chain of negative cognition as cognitive fusion and mindfulness as cognitive defusion. A parental bias for child negativity coming from a child mental disorder, such as conduct disorder ("He is bad") may, in turn, instill a negative attentional bias in the child ("I am bad"), thereby causing intergenerational transmission of negative attentional biases.

The importance of parental perception of a child's behavior, and subsequent parental preoccupations, is supported by two lines of research. First, many studies have shown that parents display dysfunctional parenting with their own child diagnosed with behavior problems, but display functional parenting when coupled with a child from another parent similarly diagnosed with behavior problems (e.g., Dumas 2005). Second, the way parents perceive the temperament of the child, more than the child's actual temperament as assessed during objective observations, is found to shape the way parents parent the child (see Bögels and Brechman-Toussaint 2006).

To conclude, parents' preoccupied attention, resulting from parents' own mental disorder or child's mental disorder, may negatively affect parenting. Mindful parenting involves open and unbiased attention directed towards the child and the parent-child interaction and thereby may improve parenting and child development.

\section{Parental Executive Functioning (Parental Reactivity)}

The third hypothesis to consider is that mindfulness improves parental executive functioning (or reduces parental reactivity) and thereby improves parenting. Parental mental disorders that are known to be associated with executive dysfunction are adult ADHD, substance abuse, autism-spectrum disorder or conduct problems, and personality disorders such as borderline personality disorder. Such disorders are likely to be associated with more reactive parenting. Also, parents of children with disorders that are related to impaired executive functioning are likely to have impaired executive functioning themselves because of the strong genetic component in externalizing disorders (e.g., Thapar et al. 1999). To illustrate, Hughes et al. (1997) found that parents of autistic spectrum children, especially fathers, had relatively poor planning skills and attention flexibility. Similarly, adults with ADHD report lower mindful awareness levels than adults without ADHD, particularly lower "acting with awareness" scores (Smalley et al. 2009). Not only do parents of children with executive functioning-related mental disorders display poorer executive function and mindful awareness themselves, there are also indications that attention and impulsivity problems in parents negatively affect the effects of parent training. That is, Sonuga-Barke et al. (2002) found maternal ADHD to be associated with lack of improvement of their preschool children diagnosed with ADHD after a parent training program. Furthermore, Harvey et al. (2003) found that mothers with the most inattention self-reports engaged in the most negative parent-child interactions after parent training for parents of ADHD children. Fathers' self-reported inattention and impulsivity were strongly associated with lax and overreactive parenting before and after the parent training, and fathers' impulsivity was related to more arguing during parent-child interactions measured before the parent training. Finally, children with impulsive or emotional symptoms may elicit more impulsive reactions from their parents to begin with, but parents with poorer executive function may have more difficulty to inhibit such reactions.

In sum, given the genetic loadings of mental disorders that are associated with executive dysfunction, the offspring of parents with poorer executive functioning have a higher chance to be predisposed to inattention, impulsivity, and reactivity. Chances are high that impulsive, reactive behavior of one member of the parent-child dyad is responded to by impulsive behavior of the other, leading to a vicious circle of negative reactivity. If impulsive parents can be taught to bring mindful awareness into the interaction with their similarly impulsive child, a circle of negative reactivity of both partners in the interaction can be prevented. Mindfulness techniques teach individuals to slow down their reactions and to be aware of their intention to act prior to taking action. In mindful parenting programs, parents are taught to take a "breathing space" (i.e., to attend to their own breathing) before responding to difficult child behaviors that may trigger impulsive reactions, thus reducing parental reactivity. Consistent with this hypothesis, mindfulness, as shown above, has been found to improve executive attention. Finally, meditation helps to bring more calm in the busy or chaotic lives of parents, which may facilitate planning and organization.

\section{Intergenerational Transmission of Parenting}

The fourth hypothesis to be explored is that mindfulness training may help to break the cycle of intergenerational continuity of parenting. Parents are assumed to, in an automatic or subconscious way, repeat dysfunctional parenting patterns they have been exposed to as a child. While this has long been theorized by psychodynamic clinicians and theorists (Fonagy and Target 2002), there is also compelling empirical evidence supporting the connec- 
tion between how parents experienced their own upbringing as children and how they parent their own children (see reviews by Puttallaz et al. 1998; van Ijzendoorn 1992, and the special section introduced by Belsky et al. 2009). In particular, a lasting role for early parenting experiences has been demonstrated (Kovan et al. 2009). Intergenerational transmission of abusive parenting (e.g., Egeland et al. 1988) even among parents who consciously attempt to be good parents, further suggests a strong link between a parent's own experienced upbringing and his/her parenting of his/ her own children.

One of the ways in which parenting may be transmitted through generations is through cognitive schemas. Parents possess "parenting schemas" that consist of information and experiences that they have learned as children, when they were parented by their own parents (Young 1994). Dysfunctional parenting schemas are activated by the emotions arising during parenting, and particularly in situations that bear emotional resemblances with past experiences in parents' childhood (e.g., a child having a tantrum activates a schema related to aggression in the family of origin). These schemas may guide parenting behavior, without parents being fully aware of it. In addition, the strong correlation between mother's own attachment schema's and their infants' attachment classification suggests a transmission of attachment style from mother to infant (van Ijzendoorn and BakermansKranenburg 1996). Particularly when under stress, parents may repeat dysfunctional and even abusive parenting patterns (Siegel and Hartzell 2004). Under stress, automatic, subconscious schemas and behaviors are activated via the amygdale without modification from higher cortical levels (LeDoux 1998).

One way of preventing intergenerational transmission of dysfunctional parenting is through applying mindfulness to parents' categorical memory. Wahler et al. (2008) have developed a method to help parents of clinically referred children enhance the objective study of their past experiences with their own parents that are activated in current parent-child stressful interactions. Parents' stories about how they themselves were parented and about how they experience parenting their own child are recorded in a structured way by having them answer four questions: (1) "Why did you bring your child to our clinic?"; (2) "What's it like to live with your child?"; (3) "When your child was younger, what do you remember about being a parent?"; and (4) "Tell me about what you can remember about life with your parents?"(Wahler et al. 2008, p. 226). Mindfulness techniques are used to help parents restructure these present and past parenting.

To conclude, mindfulness and particularly being mindful during and about emotionally intense, stressful, or painful parent-child interactions may prevent and stop intergenerational transmission of dysfunctional upbringing patterns.

\section{Self-Nourishing Attention}

The fifth hypothesis is that mindfulness, through promoting self-nourishing attention or self-compassion, improves parenting. Becoming a parent involves a shift of attention and resources from the self towards one's child, thereby reducing self-nourishing attention. Taking care of oneself while taking care of one's child may be a prerequisite for good parenting. Kabat-Zinn and Kabat-Zinn (1997) note that when parents' inner resources become depleted, they have to find effective ways to replenish them, without doing so at the expense of their children.

Self-nourishing attention may be particularly important for parents suffering from mental disorders, as they may have grown up in environments lacking in positive attention from parents. As a consequence, they may lack the ability to provide positive attention to themselves. Parents of children with mental disorders may also have greater difficulty providing themselves with self-nourishing attention, due to the increased demands and stresses of raising a child with a mental disorder (e.g., Baker-Ericzen et al. 2005). In line, Brems et al. (1993) highlighted the importance of attending to the parents' own emotional needs during behavioral parent training.

Neff (2003) defined self-compassion as being composed of three main components: being kind and understanding toward oneself in instances of hardship or perceived inadequacy rather than being harshly self-critical; perceiving one's experiences as part of the larger human experience rather than seeing them as isolating; and holding painful thoughts and feelings in mindful awareness rather than over-identifying with them. Mindfulness interventions teach participants to adopt a more accepting, nonjudgemental, and compassionate stance toward themselves (Segal et al. 2002). Siegel (2009, p. 145) suggested that "Maybe mindfulness is actually a relational process where you become your own best friend," as the social circuitry of the brain gets activated in mindfulness practice. By devoting time to the meditation practices, parents learn to devote positive attention to the self, and to begin to experience self-compassion. This may bring about a restored balance between attention for the child and selfattention and may be one of the mechanisms through which mindfulness training works for parents. Moreover, becoming more compassionate towards themselves may in turn help parents to be more accepting and compassionate towards their children, especially when their children express negative emotions.

Loving kindness meditation is a form of mindfulness practice which specifically aims to increase positive attention to the self. Within the Buddhist tradition, the goal of this meditation is to increase awareness of, and compassion for, the suffering of others; however, the 
assumption is that self-compassion is the necessary first step. The participant directs statements of compassion and well-wishes toward the self, towards a loved one, towards an emotionally neutral person, towards a difficult person, and finally towards all living beings (Salzberg 1995). Loving kindness meditation is hypothesized to increase self-nourishing attention in several ways. First, it may counteract negative biases by having participants deliberately attend to compassionate feelings towards themselves. Second, the assumption behind loving kindness meditation is that all individuals are worthy of compassion and unconditional love. Parents are taught to cultivate the intention to be compassionate and unconditionally loving towards themselves and others, regardless of how they feel about themselves or others in that moment. In this way, the practice reinforces the idea that compassion and caring for self are a given and do not have to be earned. A further assumption is that all individuals have the capacity to love and can choose to direct that love towards themselves and others. Many individuals with mental disorders experience themselves as lacking the ability to love or be loved. Practicing this meditation may help individuals be more aware of their capacity for compassion for themselves and others.

Loving kindness meditation may be particularly useful for parenting because it deliberately plays on the dynamic connection between self and other schemas. The research cited above on transmission of parenting suggest that parents' own schemas about attachment and being parented may affect the way they parent their own children, as well as how they view themselves as parents. Parents with mental disorders or with children with mental disorders may experience feelings of guilt and inadequacy about their parenting. Due to their negative bias discussed above, they may focus on negative interactions with their children, in which they feel angry or frustrated with their children, and guilty or incompetent as parents. Deliberately bringing attention to loving feelings towards their children can have a powerful emotional effect, helping parents to re-connect with their positive feelings towards their child and view themselves more positively as parents. However, these hypotheses have yet to be tested in research.

\section{Marital Functioning and Co-parenting}

Mindfulness training may affect parenting by improving marital functioning. There is strong evidence linking marital functioning with parenting and child outcomes. For example, marital quality is associated with the quality of parenting and the parent-child relationship (Erel and Burman 1995) and is negatively associated with child behavior problems (Reid and Crisafulli 1990). Furthermore, interparental conflict has been associated with a wide variety of negative physiological, behavioral, and emotional effects on children (Cummings 1994). Mindfulness training may act on three dimensions of marital functioning: marital conflict, marital satisfaction, and co-parenting, which will be discussed below.

\section{Marital Conflict}

Mindful parenting interventions are hypothesized to help reduce interparental conflict by lowering partners' emotional reactivity to each other, thereby preventing couples from a negative spiral of anger and blame during discussions of conflict. Several of the basic mindfulness techniques are hypothesized to reduce emotional reactivity: mindful listening (i.e., listening attentively in a nonreactive and non-judgemental way); acknowledging and labeling emotional states in a non-personal way, in order to avoid being swept up in a negative emotional cycle ("there is anger"); noting feelings as passing mental events; viewing partner's angry statements as "just words" rather than facts; and having the intention to act compassionately towards one's partner, even while angry. All of these techniques aim to reduce automatic, reactive responding to emotional stressful interactions, without withdrawing from the partner emotionally or physically. For example, Thich Nhat Hanh (2001) described several mindfulness-based techniques to help partners deal with anger towards each other. Partners are taught first to recognize, acknowledge, and accept their own feelings of anger, and they are taught to communicate anger to their partner in a planned, calm, non-reactive, and compassionate way, for example by scheduling an appointment to discuss conflictual issues or by writing a letter. While these communication strategies which prevent impulsive reactive responding are not specific to mindfulness, the mindfulness techniques may enable partners to remember to slow down and respond less reactively, even when emotionally triggered. Since conflict discussions often involve emotional reactions, the mindfulness practice may help couples apply what they have learned in therapy to actual conflict situations when they are emotionally aroused.

There is some preliminary support for these hypothesized mechanisms. Wachs and Cordova (2007) found that the association between mindfulness and perceived marital quality was fully mediated by skilled emotion repertoires, specifically those associated with identifying and communicating emotions, as well as the regulation of anger expression. Similarly, trait mindfulness was associated with greater capacity to respond constructively to relationship stress, lower emotional stress responses to relationship difficulties, and reduced marital conflict from pre- to posttherapy. In addition, state mindfulness was related to better 
communication quality during discussions (Barnes et al. 2007). One practical question is whether it is more effective if both partners are trained in mindfulness techniques compared with just one; but, thus far, this has not been addressed in any studies.

\section{Marital Satisfaction}

Mindfulness techniques may help foster more positive and satisfying marital relationships by increasing couples' openmindedness and flexibility and reducing criticism and rigidity. In support of this idea, people who rated themselves as high on mindfulness were also high on marital satisfaction (Burpee and Lange 2005). Furthermore, Carson et al. (2004) found that the relationship of relatively happy nondistressed couples improved after following a mindfulness-based relationship enhancement intervention. In addition, greater mindfulness practice on a given day was correlated with improved couple relationship functioning in consecutive days. Further studies are needed to elucidate the exact mechanisms underlying the improved marital relationship, and it is not yet clear whether these results would generalize to families in which one spouse or a child is suffering from a mental disorder.

Finally, mindful attention to pleasurable activities, a technique used in MBCT, can also be used to increase marital satisfaction. Deliberately pay attention to pleasurable events in the couples' relationship may help couples become more aware of pleasant feelings for each other, as well as reduce the bias towards remembering negative interactions.

\section{Co-parenting}

Co-parenting refers to the ability of parents to support and not disqualify the partner in the presence of the child, whether the partner is present or not (McHale 1994). In families who are referred to mental health care, marital dysfunction and unresolved divorce and postdivorce coparenting issues are likely to be over-represented. Disqualification of the ex-partner is particularly relevant for divorced couples, who may not often directly interact in the presence of the child but who can send disqualifying or supporting messages about the other parent to the child. Post-separation parents have been found to interact with their children with less affection and involvement and with greater punitiveness and irritability (Hetherington et al. 1982; Wallerstein and Kelly 1980). Furthermore, single mothers perceived themselves as more stressed and were observed to display more critical and controlling parenting behaviors than either maritally distressed or supported mothers (Webster-Stratton 1990). These findings suggest that postdivorce parents and single mothers may be at highest risk of experiencing parenting stress and dysfunctional parenting. Supportive co-parenting may be lowest in these groups, because of postdivorce disqualifying coparenting or lack of co-parenting due to uninvolvement of the other parent. Teaching mindfulness to postdivorce and single parents may improve supportive co-parenting even in the absence of the other parent. Conflicts with the expartner may be better resolved, and postdivorce parents may be better able to acknowledge their need to be supported as a parent and to share the sometimes difficult parenting task with their ex, and with others. One of the interesting process questions for maritally distressed parents who are still together is whether co-parenting will improve simply as a result of improvements in marital satisfaction and conflict resolution skills, or if this area needs to be specifically targeted.

\section{Mindfulness Targeting Parents}

Several mindfulness programs have targeted parents specifically, with the assumption that, if the parent becomes more mindful in general, this will improve parenting skills and the parent-child relationship. The specific goals of mindful parenting programs are to facilitate parents to bring non-judgemental, here-and-now attention, and intentionality in parenting. We will review the programs that have been developed in the context of mental health problems, and their results, with a particular focus on the six pathways of change discussed above.

Altmaier and Maloney (2007) developed a mindful parenting program to sustain emotional and physical connectedness between parents and children at a time of the parents' divorce. Although parents were from a community sample and the study was not conducted in a mental health care setting, divorce is a period in which parenting stress is high, more dysfunctional parenting is likely to be displayed by both parents, and the parent-child relationship may suffer, as was outlined earlier. Therefore, we included the study in this review. The participants were 12 parent-child dyads, where the children were 3372 months old at the time of the intervention. The program consisted of six sessions of $2.5 \mathrm{~h}$ of mindful parenting in 12 weeks, delivered in two small groups The program assists parents with identifying interactions that lead to disconnectedness with their children (e.g., criticizing, projecting anger, humiliating, and emotionally withdrawing) and replacing those interactions with intentional connectedness-focused interactions (e.g., listening, displaying affection, responding calmly, and modeling selfsoothing behaviors). Parents are made familiar with mindfulness practices, including breathing, body awareness, centering, and meditation. The idea was that, as 
parents become more mindful, they become more aware of how their responses influence their interactions with their child and learn to be more intentional in their parenting to choose ways that enhance and sustain a positive emotional connection. The parents' level of self-reported mindfulness (as measured through the Toronto Mindfulness Scale) increased significantly as a result of the intervention. However, parent-child connectedness, as measured by behavioral home observations, did not increase from preto post-assessment. Also, in contrast to expectations, parental stress, as measured by the Parental Stress Index, was not reduced by the intervention.

Taken together, this study does not support the efficacy of mindful parenting in recently divorced parents on parental stress and the parent-child relationship. Parents' evaluations, however, showed that they considered the program overall helpful and thought that it positively affected their relationship with their child. A possible explanation for the lack of effectiveness is that the program was relatively short and participants reported difficulties applying and integrating homework practice. As the divorced parents can be assumed to be under high levels of stress, they may have needed more intense in-session and homework practice to be able to reduce their parenting stress. A second, related, explanation concerns the timing of the intervention: perhaps mindfulness is more effective when given before a divorce, or longer after the divorce has taken place, so that parents have more space to work with the mindfulness practice offered. A third explanation concerns the form of the training that focused on mindfulness skills in general, rather than on the application to parenting.

Another study that applied mindfulness targeted to parents was conducted by Singh et al. (2007). They introduced a mindfulness parenting program to four mothers of children with developmental disabilities. The training was done individually and involved meditation methods and exercises through which the mothers could practice mindfulness. An important modification was that with the introduction of each practice, its application to mother-child interactions was also discussed. This makes the mindfulness in the Singh et al. (2007) study more childand parenting-oriented than that applied by Altmaier and Maloney (2007). After the 12-week-long program, the mothers were asked to practice the skills they had obtained for 52 weeks. The results showed that the children's aggressive behavior decreased during the course and especially over the following year-long practice phase, as did the number of negative mother-child interactions observed. Observed positive interactions, on the other hand, significantly increased. Furthermore, mothers' selfratings of parental satisfaction increased over time. Similar results, using similar individual single-subject designs, were obtained in three mothers of autistic children (Singh et al. 2006) and in two mothers of children with ADHD (Singh et al. 2010). These three studies offer support for a mindful parenting program in a clinical context in which mindfulness is thought together with its application to parenting a child with a developmental disability or mental disorder that is a particular challenge to parenting and the parent-child relationship and that is likely to enhance parenting stress. Note, however, that numbers are very small. The studies do not provide further information as to whether the mindful parenting program affected one of the six mechanisms described, aside from the finding that mindful parenting changed (difficult) child behaviors that are associated with the developmental disability or mental disorder of the child, such as non-compliance.

A mindful parenting course that parents follow alongside with their children has been described and evaluated by Bögels et al. (2008). Parents of clinically referred adolescents ( $n=14$, aged 11-18 years) who suffered from externalizing disorders (ADHD, oppositional-defiant or conduct disorder, and/or autism-spectrum disorder) followed an 8-week, one-group session a week of 1.5$\mathrm{h}$ mindful parenting course, while their children followed a parallel mindfulness course for themselves (in a separate group also eight sessions of $1.5 \mathrm{~h}$ ). The majority (79\%) of parents participating suffered from a mental disorder themselves: posttraumatic stress disorder, depression, ADHD, and autistic spectrum disorder. Parents were explained that their participation was needed for three reasons: (1) generalization of skills (homework monitoring, sharing of practice experiences, and role modeling), (2) to help bring calm in their families, as children with externalizing disorders enhance parenting and family stress, and (3) parents may have similar attention or impulsivity problems because of genetic similarities, and they might find the skills useful for their own life. Note that parents were told that their participation in the mindful parenting course was necessary for the adolescent to be enrolled in the child mindfulness group. Significant and substantial improvements of the combined mindful parenting and child mindfulness training were found on adolescents' personal goals (child and parent report), externalizing and internalizing symptoms (child and parent report), child self-control (parent report), attention (child performance and child report), and child social behavior (parent report). Effect sizes of change ranged from medium to very large. Moreover, parents reported large improvement on their own goals concerning their parenting and the parent-child relationship. Despite these promising results in a referred clinical group, it remains unclear whether these positive effects result from the adolescent mindfulness training, the mindful parenting training, or the combination of both.

Next, we piloted mindful parenting in a group of ten parents only ( $n=8$ mothers of whom three were divorced, 
and one parent couple) of children of mixed ages (514 years) with mixed psychopathology: $\mathrm{AD}(\mathrm{H}) \mathrm{D}$, ODD, PDD, anxiety disorders, and V-code parent-child problem (Bögels et al., in progress). Children were not involved in the course. Taking into account the heterogeneity of the group, the mindful parenting program was primarily focused on parenting in general and not on parenting a child with particular difficulties. The program consisted of an 8-week one-session a week, 3-h group meeting, and required serious homework commitment of at least $1 \mathrm{~h}$ of meditation practice a day. Each session consisted of about $2 \mathrm{~h}$ of regular MBCT/MBSR training (body scan, sitting with the breath, breathing space, seeing and hearing meditation, mindful walking, and yoga). Roughly $1 \mathrm{~h}$ of each session focused on (1) understanding the role of parental reactivity ("the low road", Siegel and Hartzell 2004) and its relatedness to stress and fatigue, own upbringing patterns, personal or relational difficulties (e.g., lack of perceived partner support), child mental health problems (e.g., behavior problems), and historically grown parent-child interaction patterns, (2) taking care of yourself as a parent, (3) non-judgmental attention for the child, (4) acceptance of the child and its difficulties, and (5) rupture and repair in the context of parenting. Roughly two third of the homework focused on regular MBSR/MBCT formal and informal meditation practice, and one third concerned homework practice bringing mindfulness into parenting. All parents except one completed the program, and session and homework compliance was excellent.

All parents except one completed a series of questionnaires before and after the program, measuring parental stress, parental rearing style, marital satisfaction, and coparenting. Results indicate that the program strongly reduced parental rejection of the child and had positive effects on autonomy encouraging parental rearing style, on reducing parental overcontrol, on increasing affection and togetherness in co-parenting and decreasing disagreements in co-parenting, on reducing parental stress, and on increasing marital satisfaction for those who were married. Results provide some preliminary support for two of the six proposed pathways of change by mindful parenting in a mental health care context: reducing parental stress and the assumed effect on parental reactivity and improving marital functioning and co-parenting. The design and small power of this pilot study prohibits further analysis of mediation of change mechanisms, but one direction of research would be to investigate whether reduction of parental stress is the mechanism through which parental reactivity towards the child is reduced. In addition, as meditation directly affects attention processes such as disengaging from negative stimuli (Ortner et al. 2007), the reduced parental rejection of the child might be a direct consequence of mindfulness practice. Furthermore, the reduced parental rejection is consistent with the finding that meditation improves executive attention (Jha et al. 2007).

Vieten and Astin (2008) considered the negative effects that prenatal stress and low mood can have on the motherinfant attachment relationship and child development, in a small randomized control trial $(n=31)$. With this in mind, they developed a mindfulness-based intervention program ("Mindful Motherhood") for to-be mothers, with the goal of improving mothers' mood and reducing stress in pregnancy. The program cultivated mindfulness of feelings, thoughts, and body, through breath awareness, body awareness meditation, and mindful hatha yoga. In addition, acceptance and observation of self were incorporated. The results showed that mothers-to-be who had participated in the mindfulness program had significantly lower state anxiety and better mood than waitlist controls. These differences did not hold in a 3-month follow-up, though, and there was no follow-up of whether the program had any effect on infant development. However, this was just a small-scale pilot study and certainly justifies further research to investigate the potential of the program. Similar studies on mothers-to-be with mental disorders still need to be conducted.

There is also preliminary work in the context of intergenerational transmission of disorder that targets the parent-infant relationship. We are in the final stages of a project that investigates how a specific child-oriented mindfulness intervention, aimed at mothers who suffer from postnatal depression or anxiety disorders, affects mother-child interaction (Stein et al., in progress). The goal of the project was to investigate whether the presentoriented, purposeful attention that mindfulness cultivates would decrease the mothers' negative, preoccupied thinking, and therefore allow the mothers to redirect their attention to their children. Piloting demonstrated that because of the often hectic nature of life with young children, asking postnatal mothers to commit to the amount of time that a typical mindfulness course involves would be too much. Therefore, we developed a 5-min long meditation practice that we asked the mothers to do at home every day for 2 weeks before they visited us in our laboratories.

The participants were randomized to either the mindfulness or the control intervention. The laboratory visit started by the mothers playing with their child, so as to obtain a baseline measure of their interaction. Next, we primed the mother to become preoccupied (by asking them to think about their worries) and then gave them either a mindfulness or a control intervention. Both were 10 min long and were presented through headphones, while a researcher played with the child to keep him/her occupied. Afterwards, the mother and child were asked to play together again, so that we could compare the mother-child interaction after the intervention. The control intervention just asked the 
mothers to think about their own thoughts. The mindfulness intervention started with a short breathing meditation, which was familiar from the home practice. The rest of the intervention was a mindful meditation focussing on the child, whom the mother could see while listening. The recording asked the mother to pay attention to the texture of the child's hair, the color of their clothes, and so on, as if they saw the child for the very first time. Participants' selfreports have indicated that, on the whole, they found the intervention pleasant, and many have stated that it has been helpful. The results of the effect of the intervention on mother-child interaction are still pending and will allow us to evaluate the effect of the intervention on a more objective measure.

Also, mindful parenting has been described in the context of prevention of psychopathology in early childhood by the enhancement of mentalizing (Fonagy 1998). Reynolds (2003) described a parent-infant group approach to enhance reflective capacity in parents. Parents develop an active observational stance, through the accumulated practice of directing quieted, patient, curious, alive attention to both infant and self and through learning to respect and follow the child's lead in contact-seeking and exploration. The parent's activity of simply slowing down enough to notice serves an empathic function. Thus, the infant's capacity to see, feel, venture into, experience, and eventually think about the textural, dimensional properties of their world (hard-soft, close-far, fast-slow) is brought to the foreground of the parent's mind for reflection. The parents, together with four to six infants that are grouped developmentally, with no more than 4 months' difference in age, make an 8-week commitment after which they may continue for as long as they find the experience useful. The average length of stay was 6 months. Results have been described in case vignettes, demonstrating parents' increased tolerance for insecurity (process 1: stress), as well as their ability to wait (process 3: executive functioning). No quantitative results were provided.

Finally, in the context of adolescent drug prevention, Duncan et al. (2009) added mindful parenting to a behavior intervention called Strengthening Families Program, for parents of early adolescents. Five core aspects of mindful parenting were added: (1) listening with full attention, (2) maintaining emotional awareness of oneself and one's child during parenting interactions, (3) practicing non-judgmental openness and receptivity when children share their thoughts and feelings, (4) regulating one's own automatic reactivity to child behaviors, and (5) adopting compassion to oneself as a parent and toward the struggles one's child faces. Five normal community families (four couples, one single mother) of five sixth-grade girls (average age, 11.5 years) participated in a 2-h program for 7 weeks. Qualitative evaluation took place in the form of a focus group. Parents reported greater awareness of how their moods affect how they react and less reactivity towards their children (process 1).

To conclude, several mindful parenting programs have been developed in the mental health or prevention context, either as a stand-alone treatment or in combination with behavior interventions. Most programs focused on parents of children with mental disorders, some on parents with mental disorders, and some on at-risk parents. There is preliminary evidence that mindful parenting programs reduce parental stress and resulting parental reactivity (process 1), reduce aspects of child mental disorder (process 2), and improve marital functioning and co-parenting (process 6). Research, however, is limited to evaluation of pilot studies without control groups other than waitlist control.

\section{Discussion}

Mindfulness is an increasingly widely used intervention for different psychological and physiological problems and illnesses. In recent years, there has also been some mindfulness work that has specifically targeted parents. Quite a number of studies suggest that attention is one of the cognitive mechanisms that is affected by mindfulness and that potentially plays a role in the different processes addressed by mindfulness for parents in clinical settings: (1) reducing parental stress; (2) reducing parental preoccupation resulting from parental and/or child psychopathology; (3) improving parental executive functioning in impulsive parents; (4) breaking the cycle of repeating dysfunctional own upbringing schemes and habits; (5) increasing selfnourishing attention; and (6) improving marital functioning and co-parenting.

The results of the studies targeting primarily parents and their parenting skills are, in principle, hopeful, particularly those that demonstrated changes in child symptomatic behavior, especially since mindful parenting does not specifically target problematic child behavior. However, it is important to keep in mind that the children of the parents in the studies had different diagnoses, varied in age, and neither study had a control group. In general, on the basis of the current small-scale studies, it is difficult to point out what the observed effects were due to. Therefore, it would be very important to conduct larger-scale, controlled studies that would have power to detect effects of treatment as well as address mediation mechanisms.

In future, it would be important to systematically investigate the effectiveness of mindfulness programs for different participant groups (with respect to child problems as well as parental problems) as well as testing specific hypotheses about the six mechanisms through which the program might work in clinical settings. This is crucial if we are to achieve a more comprehensive understanding of 
how and when to apply mindful parenting for parenting issues. This would enable us to make more precise predictions as to which target groups of parents and their children might benefit from mindful parenting and how to best tailor them to suit participants' needs.

Mindfulness may not be the most appropriate approach to all disorders, and although the currently available experiments strongly suggest that attention is implicated, this is still a very vague principle to apply when deciding on treatment approaches. Furthermore, since mindfulness does need to be adapted to suit busy parents' schedules, it would be waste of resources to use mindful parenting with groups that are unlikely to benefit from the treatment or with groups that benefit strongly from existing parent training programs. One example of a group of parents that might particularly benefit from mindful parenting is parents of children with externalizing disorders such as ADHD, who themselves suffer from attention and/or impulsivity problems. These parents are found not to benefit (enough) from behavior parent training programs.

Next steps in mindful parenting research thus include designing studies with larger participant numbers and control groups, and eventually running randomized controlled trials, for example, comparing mindful parenting to behavioral parent training. It is also necessary to investigate whether interventions affect the specific cognitive skills and aspects of behavior that mindfulness would be expected to target, such as different attentional skills. The hypothesis could be tested whether mindful parenting works primarily through changing attentional processes in parents, whereas behavioral parent training works primarily through changing parental behaviors. Also, it would be interesting to examine whether adding mindful parenting to behavioral parent training enhances the effect of the latter and what would be the best order of interventions: starting with mindful parenting, followed by behavioral parent training, starting with behavioral parent training, followed by mindful parenting, or combining both approaches in one parenting program. Finally, it is important to investigate whether the application of mindfulness to parenting is at all needed to achieve the kind of effects that mindful parenting appears to have or that the standard 8-week MBCT or MBSR program in mental health settings has similar effects on parents.

These are just a few questions that are pertinent at this point in time, when interest in mindfulness for parenting issues and children's psychological problems is increasing and we have some, but not enough, evidence that mindfulness might be an effective approach for these groups. The challenge now is to work out in more detail where the benefits lie and how to best tailor mindfulnessbased parent programs so that they suit this specific participant group both in the practical and theoretical terms.
Open Access This article is distributed under the terms of the Creative Commons Attribution Noncommercial License which permits any noncommercial use, distribution, and reproduction in any medium, provided the original author(s) and source are credited.

\section{References}

Altmaier, E., \& Maloney, R. (2007). An initial evaluation of a mindful parenting program. Journal of Clinical Psychology, 63, 1231-1238.

Barnes, S., Warren-Brown, K. W., Krusemark, E., \& Campbell, W. K. (2007). The role of mindfulness in romantic relationship satisfaction and responses to relationship stress. Journal of Marital and Family Therapy, 33, 482-500.

Baer, R. (2003). Mindfulness training as a clinical intervention: a conceptual and empirical review. Clinical Psychology: Science and Practice, 10, 125-143.

Baker-Ericzen, M. J., Brookman-Frazee, L., \& Stahmen, A. (2005). Stress levels and adaptability in parents of children with and without autism-spectrum disorder. Research \& Practice for Persons with Severe Developmental Disabilities, 30, 194-204.

Belsky, J. (1984). The determinants of parenting: a process model. Child Development, 55, 83-96.

Belsky, J., Conger, R., \& Capaldi, M. (2009). The intergenerational transmission of parenting: introduction to the special section. Developmental Psychology, 45, 1201-1204.

Bishop, S. (2002). What do we really know about mindfulness-based stress reduction? Psychosomatic Medicine, 64, 71-84.

Bögels, S. M., \& Brechman-Toussaint, M. (2006). Family factors in the aetiology and maintenance of childhood anxiety: Attachment, family functioning, rearing, and parental cognitive biases. Clinical Psychology Review, 26, 834-856.

Bögels, S. M., Sijbers, G., \& Voncken, M. (2006). Mindfulness- and task-concentration training for generalized social phobia. Journal of Cognitive Psychotherapy, 20, 33-44.

Bögels, S. M., Hoogstad, B., van Dun, L., de Schutter, S., \& Restifo, K. (2008). Mindfulness training for adolescents with externalising disorders and their parents. Behavioural and Cognitive Psychotherapy, 36, 1-17.

Brems, C., Baldwin, M., \& Baxter, S. (1993). Empirical evaluation of a self psychologically oriented parent education program. Family Relations: Interdisciplinary Journal of Applied Family Studies, 42I, 26-30.

Brown, K. W., \& Ryan, R. M. (2003). The benefits of being present: Mindfulness and its role in psychological well-being. Journal of Personality and Social Psychology, 84, 822-848.

Burpee, L. C., \& Lange, E. L. (2005). Mindfulness and marital satisfaction. Journal of Adult Development, 12, 43-51.

Cahn, B., \& Polich, J. (2006). Meditation states and traits: EEG, ERP, and neuroimaging studies. Psychological Bulletin, 132, 180-211.

Carson, J. W., Carson, K. M., Gil, K. M., \& Baucom, D. H. (2004). Mindfulness-based relationship enhancement. Behavior Therapy, $35,471-494$.

Coyne, L. W., \& Wilson, K. G. (2004). The role of cognitive fusion in impaired parenting: An RFT analysis. International Journal of Psychology and Psychological Therapy, 4, 469-486.

Crnic, K. A., Gaze, C., \& Hoffman, C. (2005). Cumulative parenting stress across the preschool period: Relations to maternal parenting and child behaviour at 5 years. Infant and Child Development, 14, 117-132.

Cummings, E. M. (1994). Marital conflict and children's functioning. Social Development, 3, 16-36.

Dumas, J. (2005). Mindfulness-based parent training: Strategies to lessen the grip of automaticity in families with disruptive children. Journal of Clinical Child and Adolescent Psychology, 34, 779-791. 
Duncan, L. G., Coatsworth, J. D., \& Greenberg, M. T. (2009). Pilot study to gauge acceptability of a mindfulness-based, familyfocused preventive intervention. Journal of Primary Prevention, 30, 605-618.

Egeland, B., Jacobvitz, D., \& Sroufe, L. A. (1988). Breaking the cycle of abuse. Child Development, 59, 1080-1088.

Erel, O., \& Burman, B. (1995). The interrelatedness of marital relations and parent-child relations: a meta-analytic review. Psychological Bulletin, 118, 108-132.

Fonagy, P. (1998). Prevention, the appropriate target of infant psychotherapy. Infant Mental Health Journal, 19, 124-150.

Fonagy, P., \& Target, M. (2002). Early intervention in the development of self-regulation. Psychoanalytic Inquiry, 22, 307-335.

Goodman, S. H., \& Gotlib, I. (2002). Children of depressed parents Mechanisms of risk and implications for treatment. Washington: American Psychological Association.

Grossman, P., Niemann, L., Schmidt, S., \& Walach, H. (2004). Mindfulness-based stress reduction and health benefits: a metaanalysis. Journal of Psychosomatic Research, 57, 35-43.

Hanh, T. (2001). Anger: Buddhist wisdom for cooling the flame. Riverhead Books, New York

Harvey, E., Danforth, J. S., McKee, T. E., Ulaszek, W. R., \& Friedman, J. L. (2003). Parenting of children with attentiondeficit/hyperactivity disorder (ADHD): The role of parental ADHD symptomatology. Journal of Attention Disorders, 7, 3142.

Hayes, S., Strosahl, K., \& Wilson, K. (1999). Acceptance and commitment therapy. New York: Guildford Press.

Hetherington, E. M., Cox, M., \& Cox, R. (1982). Effects of divorce on parents and children. In M. Lamb (Ed.), Nontraditional families. Erlbaum, Hillsdale, N. J.

Hughes, C., Leboyer, M., \& Bouvard, M. (1997). Executive functioning in parents of children with autism. Psychological Medicine, 27, 209-220.

Jha, A., Krompinger, J., \& Baime, M. (2007). Mindfulness training modifies subsystems of attention. Cognitive, Affective \& Behavioural Neuroscience, 7, 109-119.

Kabat-Zinn, J. (1990). Full catastrophe living: Using the wisdom of your body and mind to face stress, pain, and illness. New York: Dell Publishing.

Kabat-Zinn, M., \& Kabat-Zinn, J. (1997). Everyday blessings: The inner work of mindful parenting. New York: Hyperion.

Kovan, N. M., Chung, A. L., \& Sroufe, L. A. (2009). The intergenerational continuity of observed early parenting: A prospective longitudinal study. Developmental Psychology, 43, $1205-1213$.

Kristeller, J. L., Baer, R. A., \& Quillian-Wolever, R. (2006). Mindfulness-based approaches to eating disorder. In R. A. Baer (Ed.), Mindfulness and acceptance-based interventions: Conceptualization, application and empirical support. San Diego: Elsevier.

Lavee, Y., Sharlin, S., \& Katz, R. (1996). The effect of parental stress on marital quality. Journal of Family Studies, 17, 114-135.

Ledoux, J. (1998). Fear and the brain: Where have we been, where are we going? Biological Psychiatry, 44, 1229-1238.

Linehan, M. (1993). Cognitive-behavioral treatment of borderline personality disorder. New York: Guildford Press.

Ma, S., \& Teasdale, J. (2004). Mindfulness-based cognitive therapy for depression: Replication and exploration of differential relapse prevention effects. Journal of Consulting and Clinical Psychology, 72, 31-40.

MacDonald, E. E., Hastings, R. P., \& Fitzsimons, E. (2009). Psychological acceptance mediates the impact of behaviour problems of children with intellectual disabilities on fathers' psychological adjustment. Journal of Applied Research on Intellectual Disabilities, 23, 27-37.
McHale, J. P. (1994). Overt and covert co-parenting processes in the family. Family Process, 36, 183-201.

Miller-Lewis, L., Baghurst, P., Sawyer, M., Prior, M., Clark, J., Arney, F., et al. (2006). Early childhood externalizing behavior problems: Child, parenting and family-related predictors over time. Journal of Abnormal Child Psychology, 34, 891-906.

Murray, L., Kempton, C., Woolgar, M., \& Hooper, R. (1993). Depressed mothers' speech to their infants and its relation to infant gender and cognitive development. Journal of Child Psychology and Psychiatry, 34, 1083-1101.

Murray, L., Fiori-Cowley, A., Hooper, R., \& Cooper, P. (1996). The impact of postnatal depression and associated adversity on early mother-infant interactions and later infant outcomes. Child Development, 67, 2512-2526.

Neff, K. D. (2003). Self-compassion: An alternative conceptualization of a healthy attitude toward oneself. Self and Identity, 2, 85-102.

Nolen-Hoeksema, S. (1991). Responses to depression and their effects on the duration of depressive episodes. Journal of Abnormal Psychology, 100, 569-582.

Nolen-Hoeksema, S. (2000). The role of rumination in depressive disorders and mixed anxiety/depressive symptoms. Journal of Abnormal Psychology, 109, 504-511.

Ortner, S., Kilner, S., \& Zelazo, P. (2007). Mindfulness meditation and reduced emotional interference on a cognitive task. Motivation and Emotion, 31, 271-283.

Puttallaz, M., Constanzo, P. R., Grimes, D. L., \& Sherman, D. M. (1998). Intergenerational continuities and their influence on children's social development. Social Development, 7, 389427.

Reid, W. J., \& Crisafulli, A. (1990). Marital discord and child behaviour problems: A meta-analysis. Journal of Abnormal Child Psychology, 18, 105-117.

Restifo, K., \& Bögels, S. M. (2009). Family risk factors for youth depression: A family systems integration and treatment model. Clinical Psychology Review, 29, 294-316.

Reynolds, D. (2003). Mindful parenting: A group approach to enhancing reflective capacity in parents and infants. Journal of Child Psychotherapy, 29, 357-374.

Roemer, L., Orsillo, S. M., \& Salters-Pedneault, K. (2008). Efficacy of an acceptance-based behaviour therapy for generalized anxiety disorder: Evaluation in a randomized controlled trail. Journal of Consulting and Clinical Psychology, 76, 1083-1089.

Roland, E. B. (2009). Does mindfulness mediate the relationship between parental depression and negative parenting behaviors? Dissertation, University of Vermont.

Salzberg, S. (1995). Loving-kindness: The revolutionary art of happiness. Boston: Shambhala.

Sawyer, J. A. (2007). Mindful parenting, affective attunement, and maternal depression: A call for research. Graduate Student Journal of Psychology, 9, 3-9.

Segal, Z. V., Williams, M. J., \& Teasdale, J. D. (2002). Mindfulness-based cognitive therapy for depression. New York: Guilford Press.

Sethi, A. (1989). Meditation as an intervention in stress reactivity. New York: AMS Press.

Siegel, D. J. (2009). Mindful awareness, mindsight, and neural integration. The Humanist Psychologist, 37, 137-158.

Siegel, D., \& Hartzell, M. (2004). Parenting from the inside out. USA: Penguin Group.

Singh, N., Lancioni, G., Winton, A., Fisher, B. C., Curtis, W., Wahler, R., et al. (2006). Mindful parenting decreases aggression, noncompliance, and self-injury in children with autism. Journal of Emotional and Behavioral Disorders, 14, 169-177.

Singh, N., Lancioni, G., Winton, A., Singh, J., Curtis, W., Wahler, R., et al. (2007). Mindful parenting decreases aggression and increases social behavior in children with developmental disabilities. Behavior Modification, 31, 749-771. 
Singh, N., Singh, A. N., Lancioni, G. E., Singh, J., Winton, A. S. W., Singh, J., et al. (2010). Mindfulness training for parents and their children with ADHD increases the children's compliance. Journal of Child and Family Studies, 19, 157-174.

Smalley, S. L., Loo, S. K. T., Hale, S., Shrestha, A., McGough, J., Flook, L., et al. (2009). Mindfulness and attention hyperactivity disorder. Journal of Clinical Psychology, 65, 1087-1098.

Sonuga-Barke, E. J. S., Daley, D., \& Thompson, M. (2002). Does maternal ADHD reduce the effectiveness of parent training for preschool children's ADHD? Journal of the American Academy of Child and Adolescent Psychiatry, 41, 696-702.

Stein, A., Gath, D., Bucher, J., Bond, A., Day, A., \& Cooper, P. (1991). The relationship between post-natal depression and mother-child interaction. British Journal of Psychiatry, 158, 46-52.

Stein, A., Lehtonen, A., Harvey, A., Nicol-Harper, R., \& Craske, M. (2009). The influence of postnatal psychiatric disorder on child development: Is maternal preoccupation one of the key underlying processes? Psychopathology, 42, 11-21.

Tang, Y.-Y., Ma, Y., Wang, J., Fan, Y., Feng, S., Lu, Q., et al. (2007). Short-term meditation training improves attention and selfregulation. PNAS, 43, 17152-17156.

Teasdale, J., Scott, J., Moore, R., Hayhurst, H., Pope, M., \& Paykel, E. (2001). How does cognitive therapy prevent relapse in residual depression? Evidence from a controlled trial. Journal of Consulting and Clinical Psychology, 69, 347-357.

Thapar, A., Holmes, J., Poulton, K., \& Harrington, R. (1999). Genetic basis of attention deficit and hyperactivity. British Journal of Psychiatry, 174, 105-111.

Valentine, E., \& Sweet, P. (1999). Meditation and attention: A comparison of the effects of concentrative and mindfulness meditation on sustained attention. Mental Health, Religion and Culture, 2, 59-70. van Ijzendoorn, M. H. (1992). Intergenerational transmission of parenting: A review of studies in non-clinical populations. Developmental Review, 12, 76-99.

van Ijzendoorn, M. H., \& Bakermans-Kranenburg, M. J. (1996). Attachment representations in mothers, fathers, adolescents, and clinical groups: A meta-analytic search for normative data. Journal of Consulting and Clinical Psychology, 64, 821.

Vieten, D., \& Astin, J. (2008). Effects of mindfulness-based intervention during pregnancy on prenatal stress and mood: Results of a pilot study. Archives of Women's Mental Health, 11, 67-74.

Wachs, K., \& Cordova, J. V. (2007). Mindful relating: Exploring mindfulness and emotion repertoires in intimate relationships. Journal of Marital and Family therapy, 33, 464-481.

Wahler, R., Rowinski, K., \& Williams, K. (2008). Mindful parenting: An inductive search process. In L. A. Greco \& S. C. Hayes (Eds.), Acceptance and mindfulness treatments for adolescents and children: A practitioner's guide (pp. 217-235). Oakland: New Harbinger Publications.

Wallerstein, J. S., \& Kelly, J. B. (1980). Effects of divorce on the visiting father child relationship. American Journal of Psychiatry, 137, 1534-1539.

Webster-Stratton, C. (1990). Stress: A potential disruptor of parent perceptions and family interactions. Journal of Clinical Child Psychology, 19, 302-312.

Wenk-Sormaz, H. (2005). Meditation can reduce habitual responding. Alternative Therapies in Health and Medicine, 11, 32-58.

Young, J. E. (1994). Cognitive therapy for personality disorders: A schema-focussed approach. Sarasota, FL: Professional Resource Press. 